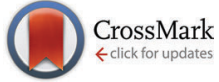

Cite this: Phys. Chem. Chem. Phys., 2015, 17, 2344

Received 24th November 2014, Accepted 5th December 2014

DOI: $10.1039 / \mathrm{c} 4 \mathrm{cp} 05466 \mathrm{k}$

www.rsc.org/pccp

\section{Hydrophobic aggregation and collective absorption of dioxin into lipid membranes: insights from atomistic simulations $\dagger$}

\author{
M. Casalegno, ${ }^{a}$ G. Raos $^{a}$ and G. Sello*b
}

\begin{abstract}
Dioxins are a highly toxic class of chlorinated aromatic chemicals. They have been extensively studied, but several molecular-level details of their action are still missing. Here we present molecular dynamics simulations of their absorption and diffusion through cell membranes. We show that, due to their hydrophobic character, dioxins can quickly penetrate into a lipid membrane, both as single molecules and as aggregates. We find clear evidence for their ability to accumulate in cell membranes. Our free energy calculations indicate that subsequent transport into the cell is unlikely to be a simple diffusive process.
\end{abstract}

Dioxins are a group of chemicals that share certain structural features and biological modes of action. ${ }^{1}$ About thirty dioxinlike compounds have been identified and they belong to three closely related families: chlorinated dibenzo-p-dioxins (CDDs), chlorinated dibenzofurans (CDFs) and certain polychlorinated biphenyls. The name dioxin is also used for the most well-studied and toxic compound in the CDD series, 2,3,7,8-tetrachlorodibenzo$p$-dioxin (TCDD, see Scheme 1).

CDDs and CDFs are not naturally occurring chemicals, but they can be unintentionally produced and introduced into the environment by a number of human activities: combustion of chlorinated compounds, chlorine bleaching of pulp and paper, certain types of chemical manufacturing and processing,

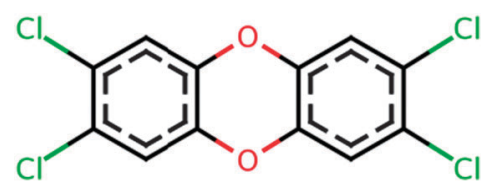

Scheme 1 Structure of TCDD.

\footnotetext{
${ }^{a}$ Dipartimento di Chimica, Materiali e Ingegneria Chimica "G. Natta",

Politecnico di Milano, Via L. Mancinelli 7, 20131 Milano, Italy

${ }^{b}$ Dipartimento di Chimica, Università degli Studi di Milano, Via C. Golgi 19,

20133 Milano, Italy. E-mail: guido.sello@unimi.it

$\dagger$ Electronic supplementary information (ESI) available: Details of the MD simulations, tests of the statistical convergence of free energy, plots of some structural descriptors. See DOI: 10.1039/c4cp05466k
}

accidents in chemical plants and other industrial activities. ${ }^{2}$ They are potent animal toxicants with the potential for producing a broad spectrum of adverse effects in humans. Due to their chemical stability and strong tendency for being absorbed by fat tissue, dioxins persist for a long time in the body. They can alter the growth and metabolism of cells, leading to adverse effects such as impaired reproduction, abnormal development, suppression of the immune system, chloracne and cancer. ${ }^{3-5}$ Based on the weight of animal and human evidence, TCDD has been classified as a "human carcinogen" under the EPA's draft guidelines, ${ }^{6,7}$ the other dioxins being "likely human carcinogens".

The mechanisms of action of TCDD have been extensively studied. TCDD and related dioxins are well established ligands for the aryl hydrocarbon receptor (AhR), ${ }^{8-10}$ a protein which modulates the transcriptional activation of many genes, including those involved in fatty acid metabolism, cell cycle regulation, immune response and xenobiotic metabolism. Binding of TCDD to AhR triggers its translocation into the nucleus and its heterodimerization with the AhR nuclear translocator protein (Arnt). ${ }^{8}$ The AhR-Arnt complex activates transcription by binding to dioxin-responsive elements, although some studies ${ }^{10}$ question the absolute requirement of AhR for transactivation of TCDD-responsive genes and hint at the existence of alternative, AhR-independent pathways. There are several other uncertainties and missing details in this picture, but the absorption and diffusion of TCDD within the cell membrane and into the cytoplasm are clearly the essential first steps. Despite the large number of studies on its toxicokinetics, ${ }^{1,6,7}$ the current understanding of how these processes occur at the molecular level is quite limited. Further progress may soon be made, thanks to recent crystallographic results on the AhR structure. ${ }^{11}$

Molecular dynamics (MD) represents a powerful computational tool, which has been used to characterize the partitioning of small organic molecules in cell membranes. ${ }^{12}$ Other studies have been conducted to investigate the accumulation and the diffusion of organic contaminants, ${ }^{13-15}$ as well as ion and drug transport. ${ }^{16-18} \mathrm{MD}$ has also been applied to study the interaction of TCDD and related compounds with inorganic ${ }^{19}$ and 
organically modified clays,${ }^{20}$ as well as ionic liquids,${ }^{21}$ in order to understand their absorption characteristics and test their effectiveness in the removal of these contaminants from the environment.

In the work reported herein, we have applied MD to simulate the absorption and diffusion of TCDD, from water into a model membrane made of 1,2-dipalmitoyl-sn-glycero-3-phosphocholine (DPPC). A force field for the description of the bonded and nonbonded interactions of TCDD was developed from suitable OPLS-AA parameters. ${ }^{22,23}$ For the DPPC membrane, we adopted a recent united-atom lipid force field, ${ }^{24}$ which was designed to be compatible with OPLS-AA. We used the TIP3P model for water. ${ }^{25}$ All simulations were carried out using the GROMACS-4.6 program suite, ${ }^{26}$ at constant temperature $(325 \mathrm{~K})$ and pressure (1.0 atm). As an input structure for the simulations, we used a pre-equilibrated bilayer containing 128 DPPC molecules in water $^{27}$ (average simulation box size: $6.4 \times 6.4 \times 12.0 \mathrm{~nm}$; the membrane is perpendicular to the $z$ axis). A variable number of TCDD molecules were placed in water, to simulate systems with different concentrations of this pollutant. Henceforth, a system containing $N$ dioxin molecules will be denoted $N$-TCDD. Further details are given in the ESI. $\dagger$

As a starting point for our investigation, we have considered the absorption dynamics in the 1-TCDD system. As shown in Fig. 1, a TCDD molecule may approach the bilayer several times within a $20 \mathrm{~ns}$ simulation (A), before a significant interaction with the membrane occurs (B). At this point, the molecule enters the membrane in less than one ns (C). Once absorbed, it locates preferentially at about $1 \mathrm{~nm}$ from the bilayer centre (D).

To gain more quantitative information on the absorption process, we performed position-restrained simulations on the 1-TCDD system. ${ }^{29-35}$ The free energy $\Delta G(z)$ and local diffusion
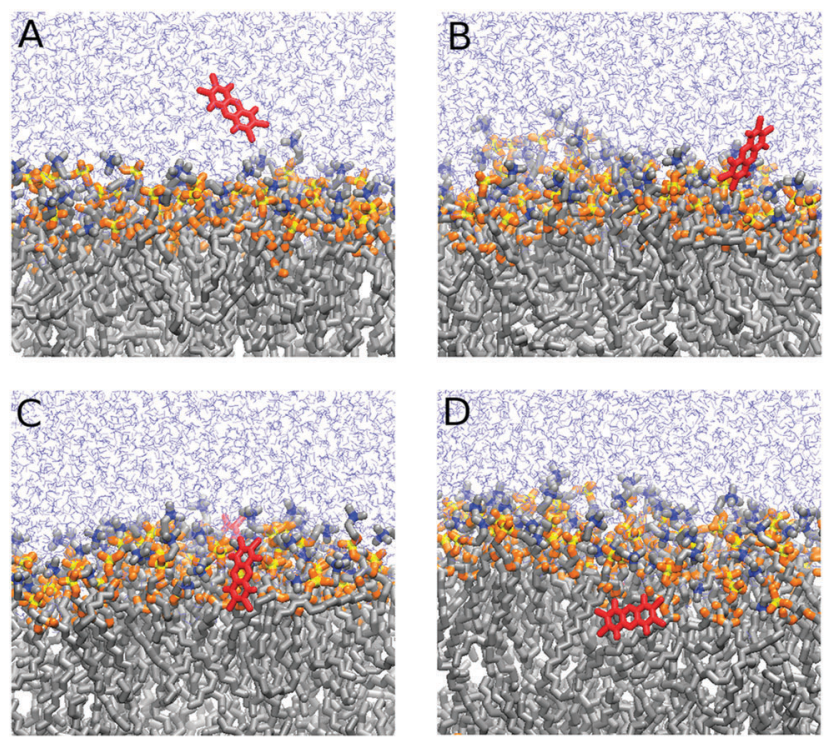

Fig. 1 Four snapshots from a 20 ns simulation of the 1-TCDD system, at (A) $2.0 \mathrm{~ns}$, (B) $12.3 \mathrm{~ns}$, (C) $12.6 \mathrm{~ns}$, and (D) $15.0 \mathrm{~ns}$. All the images from the simulations were produced with $\mathrm{VMD}^{28}$

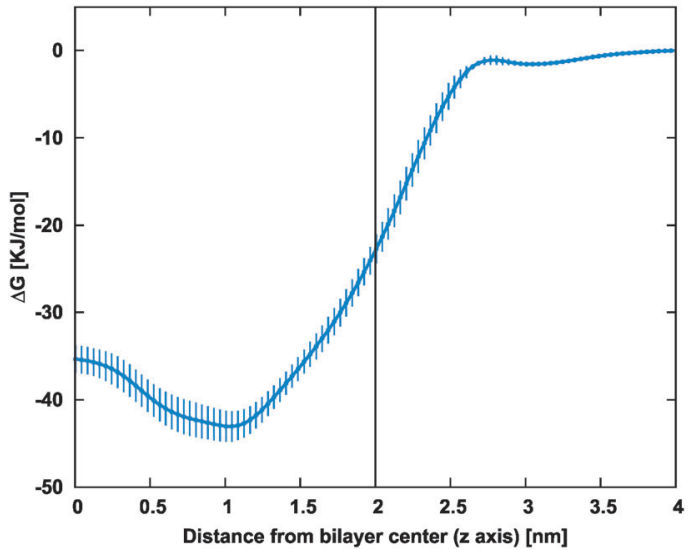

Fig. 2 Free energy profile for one TCDD molecule entering the membrane. The standard error is indicated by vertical bars. The average $z$-coordinate of the $\mathrm{P}$ atoms (about $2 \mathrm{~nm}$ from bilayer center) is indicated by the vertical line.

coefficient $D(z)$ of dioxin were evaluated via the force autocorrelation method. ${ }^{30}$ To enhance the sampling, all quantities were averaged over five independent sets of simulations, each comprising twenty equally spaced positions along the bilayer normal, from 4 to $0 \mathrm{~nm}$. Further details, including statistical tests of the convergence of the free energy, are provided in the ESI. $\dagger$ The final free energy profile, shown in Fig. 2, confirms that absorption into the membrane is a spontaneous process, with little or no barrier to the entrance of one TCDD molecule. The position of the absolute free energy minimum is consistent with the MD simulation of Fig. 1, confirming that the molecule locates preferentially away from the membrane centre. This contrasts with the results obtained for small organic molecules, ${ }^{12}$ and suggests that TCDD behaves more like $\mathrm{C}_{60}$ (ref. 13,14$)$ and pyrene, ${ }^{15}$ in this respect. The free energy change on transferring it from water $(z>4 \mathrm{~nm})$ to the bilayer $(z \approx 1 \mathrm{~nm})$ is $-43 \pm 1.8 \mathrm{~kJ} \mathrm{~mol}^{-1}$. Its absolute value is higher than that found for benzene $\left(-4.8 \mathrm{~kJ} \mathrm{~mol}^{-1}\right)^{12 a}$ and benzocaine $\left(-24.0 \mathrm{~kJ} \mathrm{~mol}^{-1}\right),{ }^{18}$ but comparable to that estimated for $\mathrm{C}_{60}\left(-35.0 \mathrm{~kJ} \mathrm{~mol}^{-1}\right) .{ }^{13}$

The permeability of a membrane, which is the truly relevant and experimentally accessible quantity, is the proportionality factor linking the steady-state flux of molecules $(J)$ to the concentration difference between its two sides:

$$
J=P\left(c_{\text {out }}-c_{\text {in }}\right)
$$

According to the inhomogeneous solubility-diffusion model, ${ }^{30,32,33}$ it can be calculated from the integral of the ratio between $\exp (\Delta G(z) / R T)$ and $D(z)$. Our final result is $P=$ $33.7 \pm 1.2 \mathrm{~cm} \mathrm{~s}^{-1}$. The current lack of experimental data on dioxin permeability prevents us from testing this value, but it can be compared with some literature data for other compounds. Using a multiscale MD simulation Orsi et al. ${ }^{33}$ calculated the permeability of a number of small molecules, obtaining values ranging from $8 \times 10^{-2} \mathrm{~cm} \mathrm{~s}^{-1}$ for acetamide to $15 \mathrm{~cm} \mathrm{~s}^{-1}$ for methyl acetate (using standard atomic level $\mathrm{MD}$ the values were from $7 \times 10^{-3}$ to $9 \mathrm{~cm} \mathrm{~s}^{-1}$ ). In another work, ${ }^{36}$ the permeability to the neutral forms of aspirin and ibuprofen were calculated to be $244 \mathrm{~cm} \mathrm{~s}^{-1}$ and $92 \mathrm{~cm} \mathrm{~s}^{-1}$, respectively. The permeabilities of 


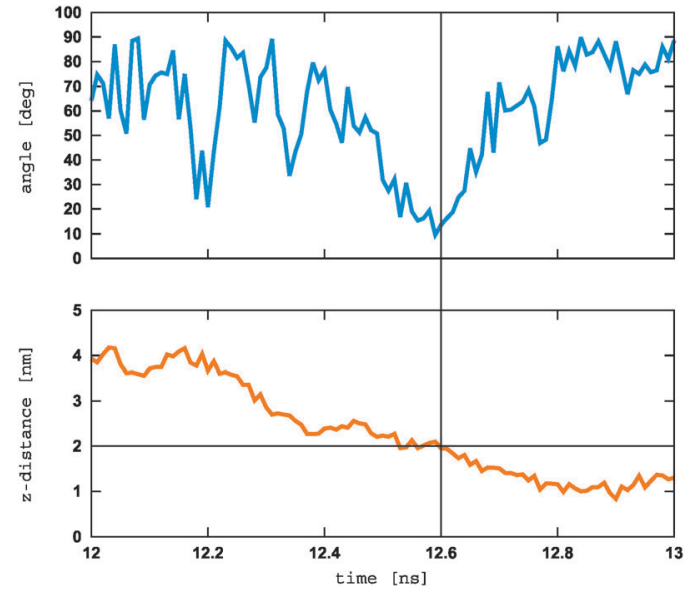

Fig. 3 Dioxin tilt angle (top) and distance from bilayer centre (bottom), versus the simulation time for the 1-TCDD system. The vertical line at $12.6 \mathrm{~ns}$ has been added to guide the eye.

cholesterol and its 4-hydroxy derivative have been estimated at $47 \mathrm{~cm} \mathrm{~s}^{-1} \cdot{ }^{37}$ Overall, a value between 5 and $50 \mathrm{~cm} \mathrm{~s}^{-1}$ appears to be reasonable for TCDD, in view of the balance between its hydrophobicity and polarity. This further confirms the tendency of dioxin to be quickly absorbed by DPPC.

The calculation of structural parameters, such as the areaper-lipid, and membrane thickness, revealed no major changes in the bilayer structure upon dioxin absorption (see ESI $\uparrow$ for details). Instead, there is a strong correlation between dioxin absorption and orientation. Fig. 3 shows the tilt angle between the TCDD molecular plane and the normal surface vector during a $20 \mathrm{~ns}$ MD simulation. It can be seen that the molecule adopts a perpendicular orientation when it enters the membrane. After the absorption, the tilt angle increases as the molecule reaches a stable configuration, with a preferentially parallel orientation (see also Fig. 1C and D).

As a next step, we have investigated systems containing $N=2,3,5$ or 10 TCDD molecules. These simulations lasted from several tens to hundreds of ns (see ESI $\dagger$ ). Due to strong hydrophobic interactions, the TCDD molecules quickly aggregate in water to form clusters. Like the single molecule, also the clusters were observed to approach the membrane surface several times before entering it. In all cases, the absorption of a whole cluster is triggered by the entrance of one molecule into the membrane. Fig. 4 shows this process for the 10-TCDD system. After the absorption of the first molecule (Fig. 4A), the remaining ones penetrate into the membrane almost sequentially. The average distance between the molecular centres-of-mass during absorption was similar to that observed in water, suggesting that the process is driven by strong intermolecular interactions among TCDD molecules. Shortly after the entrance of the last molecule (Fig. 4B), the cluster breaks up and the molecules settle on either side of the bilayer, at about $1 \mathrm{~nm}$ from its centre, in analogy with the 1-TCDD system (see Fig. 5A).

Analogous processes were observed for the smaller clusters. In some cases, we observed the clusters split into sub-units, which then were all sequentially absorbed. In all cases, the
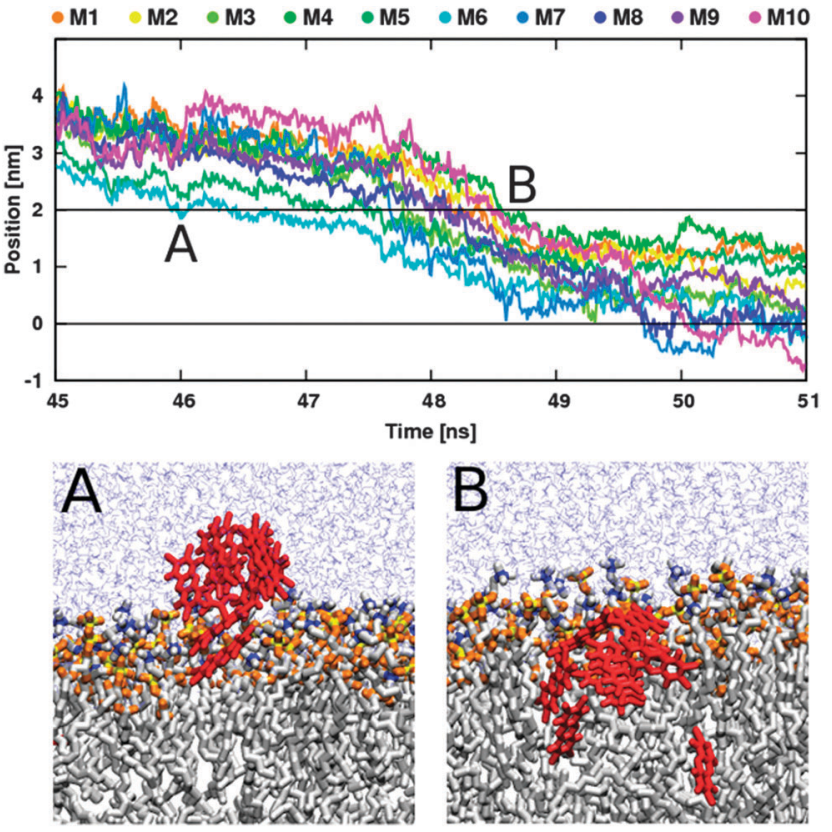

Fig. 4 Upper panel: $z$-coordinates of the molecules during a simulation of the 10-TCDD system. The horizontal lines at $z=0$ and $z \approx 2$ indicate the bilayer center and the average positions of the $\mathrm{P}$ atoms, respectively. The snapshots in the lower panel show the system at the entrance of the first (A, at $46 \mathrm{~ns})$ and last (B, at $48.6 \mathrm{~ns})$ molecule.

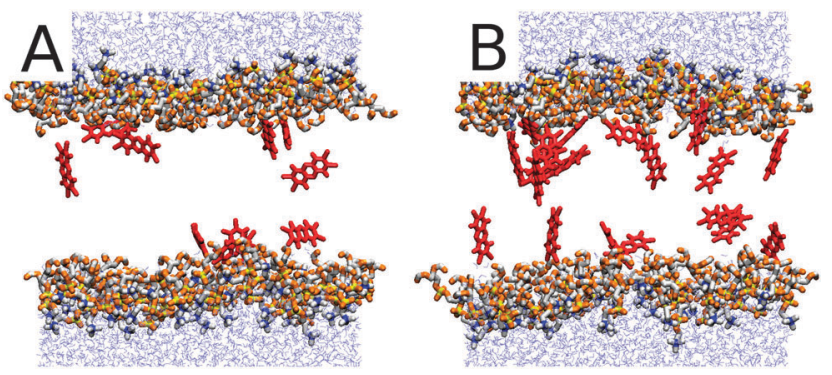

Fig. 5 Partitioning of TCDD molecules within the membrane for the 10-TCDD system (panel A) and the $10+10-$ TCDD system (panel B). The DPPC hydrocarbon chains have been omitted for clarity.

absorption of single clusters took a few nanoseconds to complete, regardless of its size. In order to assess the ability of TCDD to accumulate in DPPC bilayers, we also performed a series of simulations sequentially loading the membrane with two clusters of a given size. Even for the largest system considered $(10+10$-TCDD, meaning that 10 further molecules were added to the aqueous phase of the equilibrated 10-TCDD system), we found that the absorption of the second cluster was little affected by the TCDD molecules already present in the membrane. The distribution of the molecules within the membrane resembled that found in the 10-TCDD system (see Fig. 5B). This suggests that TCDD can accumulate in DPPC bilayers to a large extent, apparently without affecting membrane permeability.

We note that the concentration of TCDD in our simulations is higher than that established by risk assessment studies. ${ }^{1}$ Local clustering may nonetheless occur, to form stable aggregates that 
are quickly absorbed, as shown by our simulations. Although preliminary, these results suggest that cluster absorption may enhance dioxin storage in cell membranes. Considering the apparent stability of the membrane even at extreme TCDD loadings and the highly hydrophobic nature of this molecule, we believe that subsequent transport of TCDD into the cytoplasm of the cell can hardly be a diffusive process, probably requiring the mediation of dedicated transport mechanisms. Our future work will focus on some of these issues, including also the study of structurally similar molecules and more realistic membrane models. Future refinements to our modeling strategy will eventually include the adoption of other enhanced sampling techniques (e.g. temperature accelerated MD, or metadynamics), ${ }^{38-40}$ as well as the inclusion of atomic polarization to have a better description of the energetics in the passage from a polar to a non-polar environment. ${ }^{41-44}$

\section{Acknowledgements}

GS received partial funding through computational time at CINECA (LISA project ADDLIBIM) and the Università degli Studi di Milano.

\section{Notes and references}

1 U.S. EPA. Exposure and Human Health Reassessment of 2,3,7,8-Tetrachlorodibenzo-P-Dioxin (TCDD) and Related Compounds. National Academy Sciences (External Review Draft). U.S. Environmental Protection Agency, Washington, DC, EPA/600/P-00/001Cb, 2004.

2 C. Rappe, H. R. Buser and H.-P. Bosshardt, Ann. N. Y. Acad. Sci., 1979, 320, 1; E. Fattore, A. Di Guardo, G. Mariani, A. Guzzi, E. Benfenati and R. Fanelli, Environ. Sci. Technol., 2003, 37, 1503.

3 J. H. Mennear and C. C. Lee, Environ. Health Perspect., 1994, 102, 265.

4 K. Steenland, P. Bertazzi, A. Baccarelli and M. Kogevinas, Environ. Health Perspect., 2004, 112, 1265; A. Baccarelli, A. C. Pesatori, S. A. Masten, D. G. Patterson Jr, L. L. Needham, P. Mocarelli, N. E. Caporaso, D. Consonni, J. A. Grassman, P. A. Bertazzi and M. T. Landi, Toxicol. Lett., 2004, 149, 287.

5 G. Biswas, S. Srinivasan, H. K. Anandatheerthavarada and N. G. Avadhani, Proc. Natl. Acad. Sci. U. S. A., 2008, 105, 186.

6 U.S. EPA. Health Assessment Document for 2,3,7,8Tetrachlorodibenzo-P-Dioxin (TCDD) and Related Compounds (1994 Final Report). U.S. Environmental Protection Agency, Washington, DC, EPA/600/BP-92/001a (NTIS PB94205465), 1994.

7 EPA's Reanalysis of Key Issues Related to Dioxin Toxicity and Response to NAS Comments, U.S. Environmental Protection Agency, Washington, DC, EPA/600/R-10/038F, 2012, vol. 1.

8 J. P. Whitlock Jr., Chem. Res. Toxicol., 1993, 6, 754; J. Mimura and Y. Fujii-Kuriyama, Biochim. Biophys. Acta,
2003, 1619, 263; M. S. Denison, A. A. Soshilov, G. He, D. E. DeGroot and B. Zhao, Toxicol. Sci., 2011, 124, 1.

9 The AH Receptor in Biology and Toxicology, ed. R. Pohjanvirta, Wiley, Hoboken, NJ, 2012.

10 M. Nukaya, S. Moran and C. A. Bradfield, Proc. Natl. Acad. Sci. U. S. A., 2009, 106, 4923.

11 D. Wu, D. Potluri, Y. Kim and F. Rastinejad, Mol. Cell. Biol., 2013, 33, 4346.

12 D. Bemporad, J. W. Essex and C. Luttmann, J. Phys. Chem. B, 2004, 108, 4875; J. L. MacCallum and D. P. Tieleman, J. Am. Chem. Soc., 2006, 128, 125.

13 R. Qiao, A. P. Roberts, A. S. Mount, S. J. Klaine and P. C. Ke, Nano Lett., 2007, 7, 614.

14 J. Wong-Ekkabut, S. Baoukina, W. Triampo, I.-M. Tang, D. P. Tieleman and L. Monticelli, Nat. Nanotechnol., 2008, 3, 363 .

15 B. Hoff, E. Strandberg, A. S. Ulrich, D. P. Tieleman and C. Posten, Biophys. J., 2005, 88, 1818.

16 A. A. Gurtovenko, J. Anwar and I. Vattulainen, Chem. Rev., 2010, 110, 6077.

17 A. De Nicola, S. Hezaveh, Y. Zhao, T. Kawakatsu, D. Roccatano and G. Milano, Phys. Chem. Chem. Phys., 2014, 16, 5093.

18 J. J. López Cascales, S. D. Oliveira Costa and R. D. Porasso, J. Chem. Phys., 2011, 135, 135103.

19 C. Liu, H. Li, C. T. Johnston, S. A. Boyd and B. J. Teppen, Soil Sci. Soc. Am. J., 2012, 76, 110.

20 R. Zhu, W. Hu, Z. You, F. Ge and K. Tian, J. Colloid Interface Sci., 2012, 377, 328.

21 W. Pan, Y. Qi, R. Wang, Z. Han, D. Zhang and J. Zhan, Chemosphere, 2013, 91, 157.

22 W. L. Jorgensen, D. S. Maxwell and J. Tirado-Rives, J. Am. Chem. Soc., 1996, 118, 11225.

23 C. Caleman, P. J. van Maaren, M. Hong, J. S. Hub, L. T. Costa and D. van der Spoel, J. Chem. Theory Comput., 2012, 8, 61.

24 J. P. Ulmschneider and M. B. Ulmschneider, J. Chem. Theory Comput., 2009, 5, 1803.

25 W. L. Jorgensen, J. Chandrasekhar, J. D. Madura, R. W. Impey and M. L. Klein, J. Chem. Phys., 1983, 79, 926.

26 B. Hess, C. Kutzner, D. van der Spoel and E. Lindahl, J. Chem. Theory Comput., 2008, 4, 435.

27 J. Domanski, P. Stansfeld, M. S. P. Sansom and O. Beckstein, J. Membr. Biol., 2010, 236, 255.

28 W. Humphrey, A. Dalke and K. Schulten, J. Mol. Graphics, 1996, 14, 33.

29 J. Comer, K. Schulten and C. Chipot, J. Chem. Theory Comput., 2014, 10, 554.

30 S.-J. Marrink and H. J. C. Berendsen, J. Phys. Chem., 1994, 98, 4155.

31 J. Comer, K. Schulten and C. Chipot, J. Chem. Theory Comput., 2014, 10, 2710.

32 M. Paloncýová, K. Berka and M. Otyepka, J. Chem. Theory Comput., 2012, 8, 1200.

33 M. Orsi, W. E. Sanderson and J. W. Essex, J. Phys. Chem. B, 2009, 113, 12019.

34 C. Neale, C. Madill, S. Rauscher and R. Pomès, J. Chem. Theory Comput., 2013, 9, 3686. 
35 C. Neale, W. F. D. Bennett, D. P. Tieleman and R. Pomès, J. Chem. Theory Comput., 2011, 7, 4175.

36 M. B. Boggara and R. Krishnamoorti, Biophys. J., 2010, 98, 586.

37 G. Parisio, M. Stocchero and A. Ferrarini, J. Chem. Theory Comput., 2013, 9, 5236.

38 Y. Zhang and G. A. Voth, J. Chem. Theory Comput., 2011, 7, 2277.

39 M. Minozzi, G. Lattanzi, R. Benz, M. P. Costi, A. Venturelli and P. Carloni, PLoS One, 2011, 6, 1.
40 Z. Ghaemi, M. Minozzi, P. Carloni and A. Laio, J. Phys. Chem. B, 2011, 116, 8714 .

41 I. Vorobyov, W. F. D. Bennett, D. P. Tieleman, T. W. Allen and S. Noskov, J. Chem. Theory Comput., 2012, 8, 618.

42 J. P. M. Jämbeck and A. P. Lyubartsev, Phys. Chem. Chem. Phys., 2013, 15, 4677.

43 J. P. M. Jämbeck, F. Mocci, A. P. Lyubartsev and A. Laaksonen, J. Comput. Chem., 2013, 34, 187.

44 J. W. Ponder and D. A. Case, Adv. Protein Chem., 2003, 66, 27. 